\title{
HER 2/neu protein expression in gastric cancer is associated with poor survival
}

\author{
S.D. XIE ${ }^{1 *}$, C.Y. XU ${ }^{1,3^{*}}$, J.G. SHEN ${ }^{1}$, Z.N. JIANG ${ }^{2,3}$, J.Y. SHEN ${ }^{1}$ and L.B. WANG ${ }^{1}$ \\ Departments of ${ }^{1}$ Surgical Oncology, and ${ }^{2}$ Pathology, Sir Run Run Shaw Hospital; \\ ${ }^{3}$ Sir Run Run Shaw Institute of Clinical Medicine, Key Laboratory of Biotherapy of Zhejiang Province, \\ Zhejiang University School of Medicine, Hangzhou 310016, P.R. China
}

Received April 14, 2009; Accepted August 19, 2009

DOI: 10.3892/mmr_00000196

\begin{abstract}
The presence of HER 2/neu has been reported in gastric cancer, but its impact on patient survival remains unclear. The purpose of this study was to investigate the expression of HER 2/neu in gastric cancer. A total of 218 paired resected gastric cancer and corresponding normal specimens were collected. HER 2/neu protein expression was assessed by immunohistochemical staining. The correlation between HER 2/neu expression and patient clinicopathological parameters was evaluated and the prognostic significance of HER 2/neu expression was assessed by univariate and multivariate analyses. Forty-one out of 218 (18.8\%) gastric cancer specimens showed HER 2/neu-positive expression. No relationship was found between membranous HER 2/neu expression and clinicopathological parameters. However, HER $2 /$ neu expression was correlated with poorer overall survival $(p<0.001)$. In multivariate analysis, HER $2 /$ neu expression was a significant independent prognostic predictor of gastric cancer $(\mathrm{p}<0.001)$, and was associated with poor survival in gastric cancer patients. These data indicate that HER 2/neu may play a major role in the therapeutic management of gastric cancer.
\end{abstract}

\section{Introduction}

Gastric cancer is the second leading cause of cancer-related death worldwide (1). Surgery remains the mainstay of any curative treatment. Unfortunately, in advanced gastric cancer, currently available treatments including surgery, chemotherapy and radiotherapy have limited success. Since surgery is an insufficient treatment for most patients, developing active drugs against advanced gastric cancer is required $(2,3)$.

Correspondence to: Dr L.B. Wang, Department of Surgical Oncology, Sir Run Run Shaw Hospital, Zhejiang University School of Medicine, Hangzhou 310016, P.R. China

E-mail: wanglinbo@medmail.com.cn

${ }^{*}$ Contributed equally

Key words: gastric cancer, HER 2/neu, prognosis
It has been reported that trastuzumab has significant antitumor activity related to HER 2/neu expression in gastric cancer cells and xenograft models (4-6). This finding has prompted investigation of the potential clinical benefits of trastuzumab in gastric cancer. However, conflicting results have been obtained in studies on HER 2/neu and its relationship with prognosis in gastric cancer. Some studies have reported that HER 2/neu overexpression is a poor prognostic factor for gastric cancer (7-10), while others have failed to find any association between HER 2/neu overexpression and prognosis $(11,12)$. This discrepancy indicates that there may be factors othen than HER 2/neu expression levels influencing the effect of trastuzumab in gastric cancer. Accurate assessment of HER $2 /$ neu status is therefore essential to determine which patients may benefit from trastuzumab treatment.

The purpose of the present study was to examine the expression status of HER 2/neu in gastric cancer and to evaluate whether HER 2/neu expression level is correlated with clinicopathological parameters and total prognosis or gender.

\section{Materials and methods}

Case selection. Specimens were obtained from 218 patients who underwent curative resection (R0) of gastric cancer at the Department of Surgical Oncology of Sir Run Run Shaw Hospital, Zhejiang University College of Medicine, between July 1999 and August 2006. Informed consent was obtained from all patients, and the study was conducted according to the guidelines of the Hospital Ethics Committee. The patients comprised 153 males and 65 females, aged 26 to 81 years (mean 56.8 years). No patient received anti-cancer treatment prior to surgery. The correlation between the expression of HER 2/neu and clinicopathologic parameters including age, gender, differentiation, location and pTNM pathological classification according to the International Union against Cancer (UICC) were evaluated.

Immunohistochemistry. Immunohistochemical analysis for HER 2/neu expression was performed on formalin-fixed paraffin-embedded sections of surgical specimens. The slides were deparaffinized in xylene and rehydrated in gradient ethanol solution. Endogenous peroxidase was blocked with $0.3 \% \mathrm{H}_{2} \mathrm{O}_{2}$ in methanol for $10 \mathrm{~min}$. The slides were immersed 

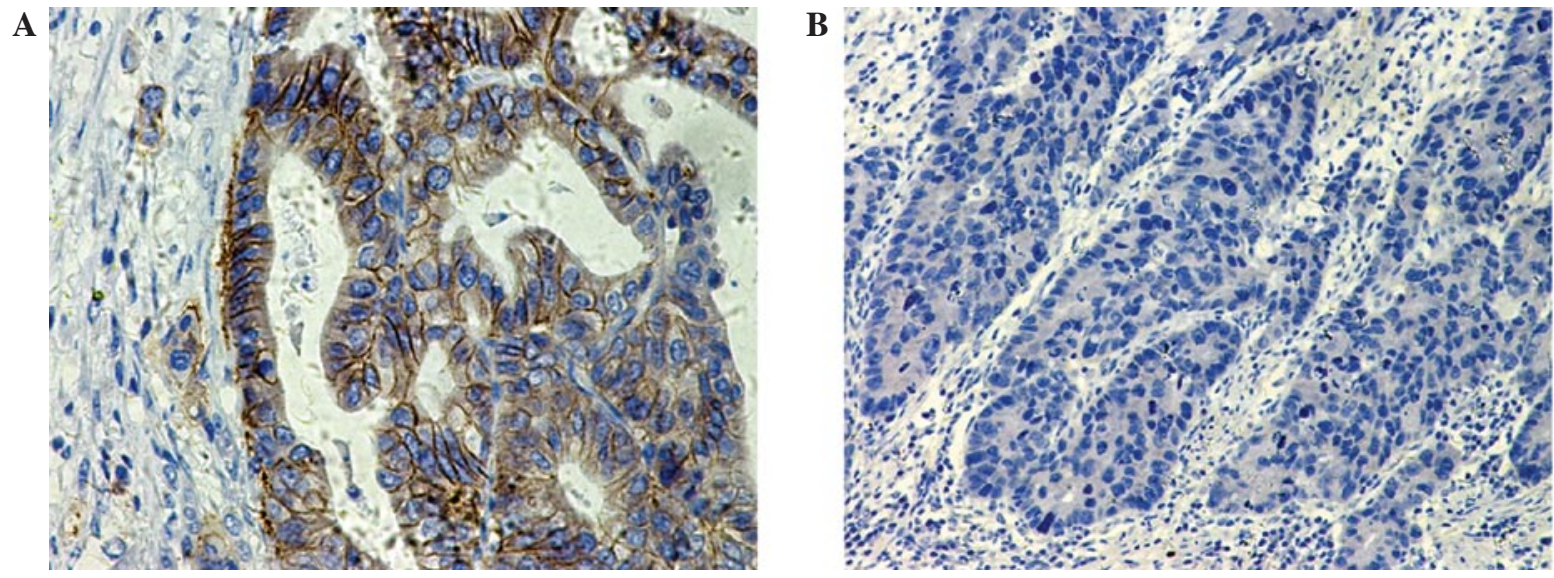

Figure 1. Immunohistochemical analysis of HER 2/neu expression in gastric cancer. (A) Strong complete cell membrane staining was observed in $>10 \%$ of tumor cells. (B) No cells were stained in the cell membranes of the gastric cancer tissue.

in $10 \mathrm{mM}$ citric buffer ( $\mathrm{pH}$ 6.0) with heating for $15 \mathrm{~min}$ for antigen retrieval, then cooled at room temperature for $20 \mathrm{~min}$ and washed with phosphate-buffered saline (PBS). Nonspecific binding was blocked by pre-incubation with $10 \%$ fetal calf serum in PBS with $0.01 \%$ sodium azide, then the slides were incubated in a humid chamber for $1 \mathrm{~h}$ with antibody against HER 2/neu HercepTest Kit (Dako Cytomation, Denmark). After washing three times in PBS, the slides were incubated with the EnVision-HRP complex (undiluted; Dako) for $60 \mathrm{~min}$, then visualized with diaminobenzidine (Dako) and counterstained with hematoxylin. For substitute negative controls, the primary antibody was replaced with PBS. Positive controls included breast cancer tissue known to exhibit high levels of marker.

The slides were examined and scored independently to avoid subjective bias by two experienced pathologists. Evaluation of the results was performed according to the criteria recommended by the manufacturer by assigning a score of 0 to $3+$. Scores were defined as follows: 0 , no staining or membrane staining in $<10 \%$ of the tumor cells; $1+$, faint/barely perceptible membrane staining in $>10 \%$ of the tumor cells, cells stained in part of the membrane; $2+$, weak to moderate staining of the entire membrane in $>10 \%$ of the tumor cells; $3+$, strong staining of the entire membrane in $>10 \%$ of the tumor cells. Specimens with scores of 0 and $1+$ were regarded as being HER 2/neu-negative, while scores of 2+ and 3+ indicated positive expression of HER 2/neu.

Follow-up. Patients underwent follow-up until death or until the date of the last follow-up on November 30, 2007. During the follow-up period, 52 patients $(23.9 \%)$ succumbed to the disease. The median follow-up interval was 51.1 months (range 6-127 months).

Statistical analysis. Statistical analysis was conducted using the statistical program SPSS 15.0 for Windows (SPSS, Chicago, IL, USA). Pre-treatment characteristics were analyzed using the 2-tailed $\chi^{2}$ test. The 2-tailed t-test was used to evaluate the correlation between HER 2/neu expression and clinicopathologic parameters. Univariate analysis of patient survival was performed using the Kaplan-Meier method. Survival curves

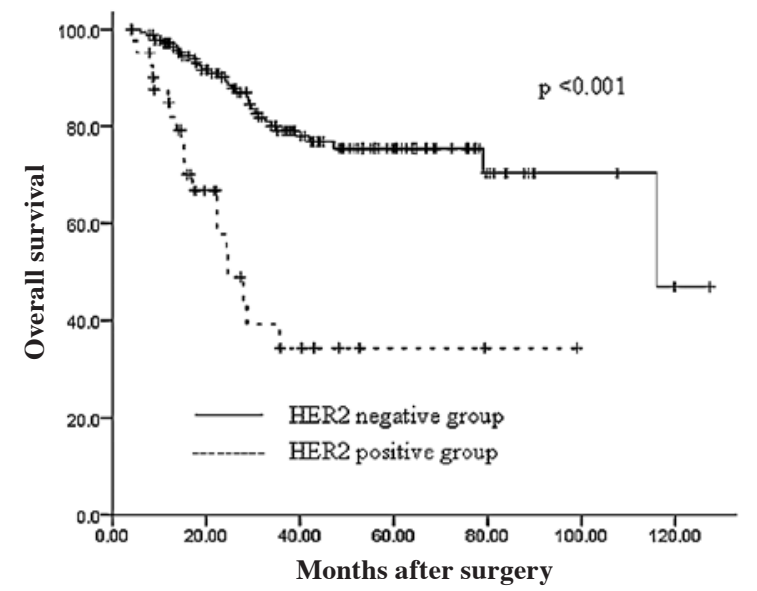

Figure 2. Overall survival. The 5-year overall survival rates were $70.6 \%$ for the HER 2/neu-negative expression group and $34.3 \%$ for the HER 2/neu-positive expression group $(\mathrm{p}<0.001)$.

were compared using the log-rank test. Multivariate analysis was performed using logistic regression and Cox's proportional hazard model. The accepted level of significance was set at $\mathrm{p}<0.05$.

\section{Results}

HER 2/neu expression is correlated with clinicopathologic parameters. The 218 gastric cancer tissue specimens were examined for the presence of HER 2/neu, determined by immunohistochemistry. Of these specimens, 41 (18.8\%) exhibited HER 2/neu-positive expression (Fig. 1). HER 2/neu expression was correlated with tumor differentiation and location. There were no differences between the groups in terms of gender, tumor size, differentiation and location.

Survival analysis. According to univariate analysis, HER 2/ neu-positive expression was associated with poorer overall survival in gastric cancer. The 5-year overall survival rates were $70.6 \%$ in the HER 2/neu-negative expression group and $34.3 \%$ in the HER 2/neu-positive expression group ( $<<0.001$; Fig. 2). In multivariate analysis, HER $2 /$ neu expression was the independent predictor (hazard ratio 6.923; p<0.001) (Table II). 
Table I. Comparison of clinicopathologic features in gastric cancer.

\begin{tabular}{|c|c|c|c|}
\hline & Negative $(n=177)$ & Positive $(n=41)$ & p-value \\
\hline Age & 55.8 & 60.8 & 0.019 \\
\hline Gender & & & 0.131 \\
\hline Male & $120(67.8)$ & $33(80.5)$ & \\
\hline Female & $57(32.2)$ & $8(19.5)$ & \\
\hline Location & & & 0.001 \\
\hline Upper body & $55(31.1)$ & $12(29.3)$ & \\
\hline Lower or middle body & $122(68.9)$ & $29(70.7)$ & \\
\hline Tumor differentiation & & & 0.476 \\
\hline Differentiated & $65(36.7)$ & $18(43.9)$ & \\
\hline Undifferentiated & $112(63.3)$ & $23(56.1)$ & \\
\hline Tumor size (mm) & 4.9 & 5.1 & 0.624 \\
\hline Serosal invasion & & & 0.851 \\
\hline No & $55(31.1)$ & $13(31.7)$ & \\
\hline Yes & $122(68.9)$ & $28(68.3)$ & \\
\hline Nodal involvement & & & 0.482 \\
\hline No & $72(40.7)$ & $14(34.2)$ & \\
\hline Yes & $105(59.3)$ & $27(65.8)$ & \\
\hline
\end{tabular}

Table II. Results of stepwise multivariate analysis for prognostic factors $(n=104)$.

\begin{tabular}{lccr}
\hline & \multicolumn{2}{c}{ Overall survival } & \multicolumn{2}{c}{ p5\% CI } & p-value \\
\cline { 2 - 3 } Variable & Risk ratio & $(2.125-22.552)$ & $<0.001$ \\
\hline Her 2/neu (positive vs. negative) & 6.923 & $(2.459-26.833)$ & 0.001 \\
Serosal invasion (yes vs. no) & 8.124 & $(1.074-1.176)$ & 0.021 \\
Node involvement & 1.124 & $(1.005-1.057)$ & 0.814 \\
Age & 1.031 & & 0.624 \\
Gender (male vs. female) & & & 0.596 \\
Location (middle vs. upper body) & & & 0.477 \\
Tumor (differentiated vs. undifferentiated) & & & \\
Tumor size & & & \\
\hline
\end{tabular}

${ }^{a}$ Cox proportional hazards model. HR, hazards ratio; 95\% CI, 95\% confidence interval; mLNs, metastatic lymph nodes; serosal invasion, pathologic tumor classification; node involvement, lymph node status. Variables entered into the regression model were HER 2/neu, serosal invasion, mLNS, age, tumor size, histologic type, gender and tumor location. Age, tumor size and number of mLNs were consecutive variables; the remaining variables were categorized.

\section{Discussion}

The HER $2 /$ neu protein is a $185-\mathrm{kDa}$ transmembrane tyrosine kinase (TK) receptor and a member of the epidermal growth factor receptor (EGFR) family $(13,14)$. Overexpression of HER 2/neu protein in gastric cancer determined using immunohistochemistry was first described in 1986 (15). Overexpression of the HER 2/neu receptor is detected in 9-38\% of human gastric cancer patients $(16,17)$. In our data, overexpression of the HER 2/neu protein occurred in 41 of 218 (18.8\%) gastric cancer specimens as determined by immunohistochemistry, which is concordant with previous reports $(18,19)$.
The role of HER 2/neu as a prognostic factor in gastric cancer is controversial $(8,9)$. Reports have suggested that HER 2/neu expression is an important prognostic factor $(20,21)$. In this study, HER 2/neu expression was examined in 218 curative resection (R0) gastric cancer cases. HER 2/neu was revealed to have a powerful adverse prognostic effect, and was an independent significant predictive factor for survival. This suggests that HER 2/neu may act as a prognostic parameter in gastric cancer.

At present, there are few therapeutic options available for gastric cancer. Our findings suggest that trastuzumab has significant anti-tumor activity in gastric cancer cases positive for HER 2/neu expression. 
In conclusion, the expression of HER 2/neu was associated with poor prognosis in gastric cancer. Consequently, HER $2 /$ neu is a potential molecular marker for predicting patient outcome in gastric cancer patients.

\section{References}

1. Hohenberger P and Gretschel S: Gastric cancer. Lancet 362: 305-315, 2003.

2. Green D, Ponce de Leon S, Leon-Rodriguez E and SosaSanchez R: Adenocarcinoma of the stomach: univariate and multivariate analysis of factors associated with survival. Am J Clin Oncol 25: 84-89, 2002.

3. Msika S, Benhamiche AM, Jouve JL, Rat $\mathrm{P}$ and Faivre J: Prognostic factors after curative resection for gastric cancer. A population-based study. Eur J Cancer 36: 390-396, 2000.

4. Kim SY, Kim HP, Kim YJ, et al: Trastuzumab inhibits the growth of human gastric cancer cell lines with HER 2 amplification synergistically with cisplatin. Int J Oncol 32: 89-95, 2008.

5. Gong SJ, Jin CJ, Rha SY and Chung HC: Growth inhibitory effects of trastuzumab and chemotherapeutic drugs in gastric cancer cell lines. Cancer Lett 214: 215-224, 2004.

6. Fujimoto-Ouchi K, Sekiguchi F, Yasuno H, Moriya Y, Mori K and Tanaka Y: Antitumor activity of trastuzumab in combination with chemotherapy in human gastric cancer xenograft models. Cancer Chemother Pharmacol 59: 795-805, 2007.

7. Allgayer H, Babic R, Gruetzner KU, Tarabichi A, Schildberg FW and Heiss MM: c-erbB-2 is of independent prognostic relevance in gastric cancer and is associated with the expression of tumorassociated protease systems. J Clin Oncol 18: 2201-2209, 2000.

8. Uchino S, Tsuda $\mathrm{H}$, Maruyama $\mathrm{K}$, et al: Overexpression of c-erbB-2 protein in gastric cancer. Its correlation with long-term survival of patients. Cancer 72: 3179-3184, 1993.

9. Mizutani T, Onda M, Tokunaga A,Yamanaka N and SugisakiY: Relationship of c-erbB-2 protein expression and gene amplification to invasion and metastasis in human gastric cancer. Cancer 72: 2083-2038, 1993

10. Tanner M, Hollmen M, Junttila TT, et al: Amplification of HER 2 in gastric carcinoma: association with Topoisomerase IIa gene amplification, intestinal type, poor prognosis and sensitivity to trastuzumab. Ann Oncol 16: 273-278, 2005.
11. Hilton DA and West KP: c-erbB-2 oncogene product expression and prognosis in gastric carcinoma. J Clin Pathol 45: 454-456, 1992.

12. Kolodziejczyk P, Yao T, Oya M, Nakamura S, Utsunomiya T, Ishikawa T and Tsuneyoshi M: Long-term follow-up study of patients with gastric adenomas with malignant ransformation. An immunohistochemical and histochemical analysis. Cancer 74: 2896-2907, 1994.

13. Akiyama T, Sudo C, Ogawara H, Toyoshima $\mathrm{K}$ and Yamamoto T: The product of the human c-erbB-2 gene: a 185-kilodalton glycoprotein with tyrosine kinase activity. Science 232: 1644-1646, 1986.

14. Coussens L, Yang-Feng TL, Liao YC, et al: Tyrosine kinase receptor with extensive homology to EGF receptor shares chromosomal location with neu oncogene. Science 230: 1132-1139, 1985.

15. Sakai K, Mori S, Kawamoto T, et al: Expression of epidermal growth factor receptors on normal human gastric epithelia and gastric carcinomas. J Natl Cancer Inst 77: 1047-1052, 1986.

16. Hofmann M, Stoss O, Shi D, Büttner R, et al: Assessment of a HER 2 scoring system for gastric cancer: results from a validation study. Histopathology 52: 797-805, 2008.

17. Gravalos $\mathrm{C}$ and Jimeno A: HER 2 in gastric cancer: a new prognostic factor and a novel therapeutic target. Ann Oncol 19: 1523-1529, 2008

18. Matsubara J, Yamada Y, Hirashima Y, et al: Impact of insulin-like growth factor type 1 receptor, epidermal growth factor receptor, and HER 2 expression on outcomes of patients with gastric cancer. Clin Cancer Res 14: 3022-3029, 2008.

19. Park DI, Yun JW, Park JH, et al: HER 2/neu amplification is an independent prognostic factor in gastric cancer. Dig Dis Sci 51: 1371-1379, 2006.

20. Tateishi M, Toda T, Minamisono Y, et al: Clinicopathological significance of c-erbB-2 protein expression in human gastric carcinoma. Surg Oncol 49: 209-212, 1992.

21. Sasano H, Date F, Imatani A, et al: Double immunostaining for c-erbB-2 and p53 in human stomach cancer cells. Hum Pathol 24: 584-589, 1993. 